PHYSICAL REVIEW B 93, 159905(E) (2016)

\title{
Publisher's Note: An artificial Rb atom in a semiconductor with lifetime-limited linewidth [Phys. Rev. B 92, 245439 (2015)]
}

Jan-Philipp Jahn, Mathieu Munsch, Lucas Béguin, Andreas V. Kuhlmann, Martina Renggli, Yongheng Huo, Fei Ding, Rinaldo Trotta, Marcus Reindl, Oliver G. Schmidt, Armando Rastelli, Philipp Treutlein, and Richard J. Warburton (Received 6 April 2016; published 15 April 2016)

DOI: 10.1103/PhysRevB.93.159905

This paper was published online on 28 December 2015 with an omission in the Acknowledgments. The Acknowledgment section should read as

"We acknowledge financial support from NCCR QSIT and the European Union Seventh Framework Programme 209 (FP7/2007-2013) under Grant Agreement No. 601126210 (HANAS). We thank M. Rakher and N. Sangouard for fruitful discussions.

J.-P.J., M.M., and L.B. made equal contributions to this work.”

The Acknowledgments have been corrected as of 22 March 2016. The Acknowledgments are incorrect in the printed version of the journal. 